Les applications micro-

\section{fluidiques à haut débit visent}

à miniaturiser un laboratoire

d'analyse à l'échelle de

quelques centimètres carrés, afin d'améliorer la rapidité

et la sensibilité des mesures. Dans ce contexte, I'utilisation de gouttelettes produites en flux continu s'avère une stratégie prometteuse permettant de manipuler des volumes allant du picolitre au nanolitre avec une grande répétabilité, ces gouttelettes faisant office de

« microtubes à essai ».

Nous discutons ici de cette approche, la « microfluidique digitale », qui nécessite de comprendre et maîtriser l'écoulement de gouttes au sein de circuits hydrodynamiques.

Nous nous intéressons tout particulièrement à la dynamique riche et complexe de ces

systèmes, qui peuvent servir de modèles pour comprendre la circulation d'objets discrets dans des réseaux, c'est-à-dire de problèmes liés au trafic.

\title{
Comment circulent des gouttes dans un laboratoire sur puce?
}

Pascal Panizza, Axelle Amon et Laurent Courbin (laurent.courbin@univ-rennes1.fr) Institut de Physique de Rennes, UMR CNRS 6251, Campus Beaulieu, Université Rennes 1, 35042 Rennes

Les techniques microfluidiques offrent des capacités uniques pour manipuler des fluides contraints géométriquement à des petites échelles de longueur, submillimétriques. Cette classe d'écoulements et leurs propriétés sont largement exploitées dans la nature. Ainsi, ces écoulements sont cruciaux pour l'organisme, puisque le réseau de la circulation du sang fait intervenir des capillaires sanguins de petits diamètres, de l'ordre de dix microns. Dans les plantes, le transport de l'eau vers les feuilles s'effectue sous l'action de forces capillaires qui agissent aux petites échelles. Certains scarabées (Hemisphaerota cyanea) profitent de la capillarité pour générer des forces de traction considérables ( 150 fois leurs poids) qui leur permettent de se défendre en adhérant à une surface.

Ces écoulements reposent sur des concepts de physique qui sont pour la plupart connus depuis longtemps. On peut citer la célèbre loi d'ascension capillaire formulée par J. Jurin au $18^{\mathrm{e}}$ siècle, ou bien celle de J.-L.-M. Poiseuille (1844), qui décrit l'écoulement laminaire d'un fluide visqueux dans un conduit cylindrique. Dans les laboratoires et l'industrie, toutefois, la microfluidique est un domaine jeune, en plein essor, qui a bénéficié de l'émergence il y a une vingtaine d'années dans le groupe de G. M. Whitesides de l'Université de Harvard, de la lithographie douce.

Cette technologie, peu onéreuse et rapide à mettre en œuvre, permet de fabriquer des dispositifs microfluidiques en polymère transparent (fig. 1) avec une grande versatilité de motifs et une très bonne résolution spatiale (quelques microns). Les dimensions d'un canal microfluidique (hauteur, largeur) étant de l'ordre de 10 à $100 \mu \mathrm{m}$, ces dispositifs permettent de manipuler des volumes de fluides extrêmement petits. Ces spécificités ont imposé la microfluidique comme l'outil idéal pour développer des techniques de criblage à haut débit en biochimie, en génétique, ou en protéomique (science qui étudie la structure et la fonction des protéines).

La microfluidique présente donc un intérêt majeur pour diverses applications, mais aussi pour les sciences fondamentales. En effet, pour les dimensions considérées et les vitesses de fluides mises en jeu, de $1 \mu \mathrm{m} / \mathrm{s}$ à $1 \mathrm{~cm} / \mathrm{s}$, les écoulements monophasiques sont laminaires et peuvent donc être décrits en utilisant une analogie entre électricité linéaire et microfluidique (voir encadré 1). Les applications requièrent toutefois l'utilisation de plusieurs fluides non miscibles, par exemple des gouttes d'un fluide s'écoulant dans un autre fluide : il est généralement moins aisé de décrire les écoulements dans ce cas, et des dynamiques riches et complexes peuvent alors émerger comme nous allons l'illustrer ici.

\section{Une usine à gouttes dans la paume de la main!}

Une approche de la microfluidique largement exploitée pour, par exemple, fabriquer des matériaux tels que des émulsions, est basée sur la production, une à une, de gouttelettes d'un fluide dispersé dans un autre fluide non miscible, à des fréquences de l'ordre de 1 à 1000 gouttelettes par seconde. La microfluidique digitale, qui repose sur cette approche, permet un contrôle inégalé des propriétés physico-chimiques des gouttes et de leur volume, du picolitre au nanolitre. On dispose de diverses géométries pour les produire, comme une jonction en $\mathrm{T}$ dans laquelle sont injectées les phases dispersées et continues, ou une géométrie de pincement hydrodynamique ("flow focusing device" en anglais) (fig. 2). Ces modules de production génèrent des trains unidimensionnels et périodiques de gouttes monodisperses confinées, qui circulent à vitesse constante $v \sim 0,1$ à $10 \mathrm{~mm} / \mathrm{s}$. En pratique, on intègre divers modules sur une même puce, chacun de ces modules 


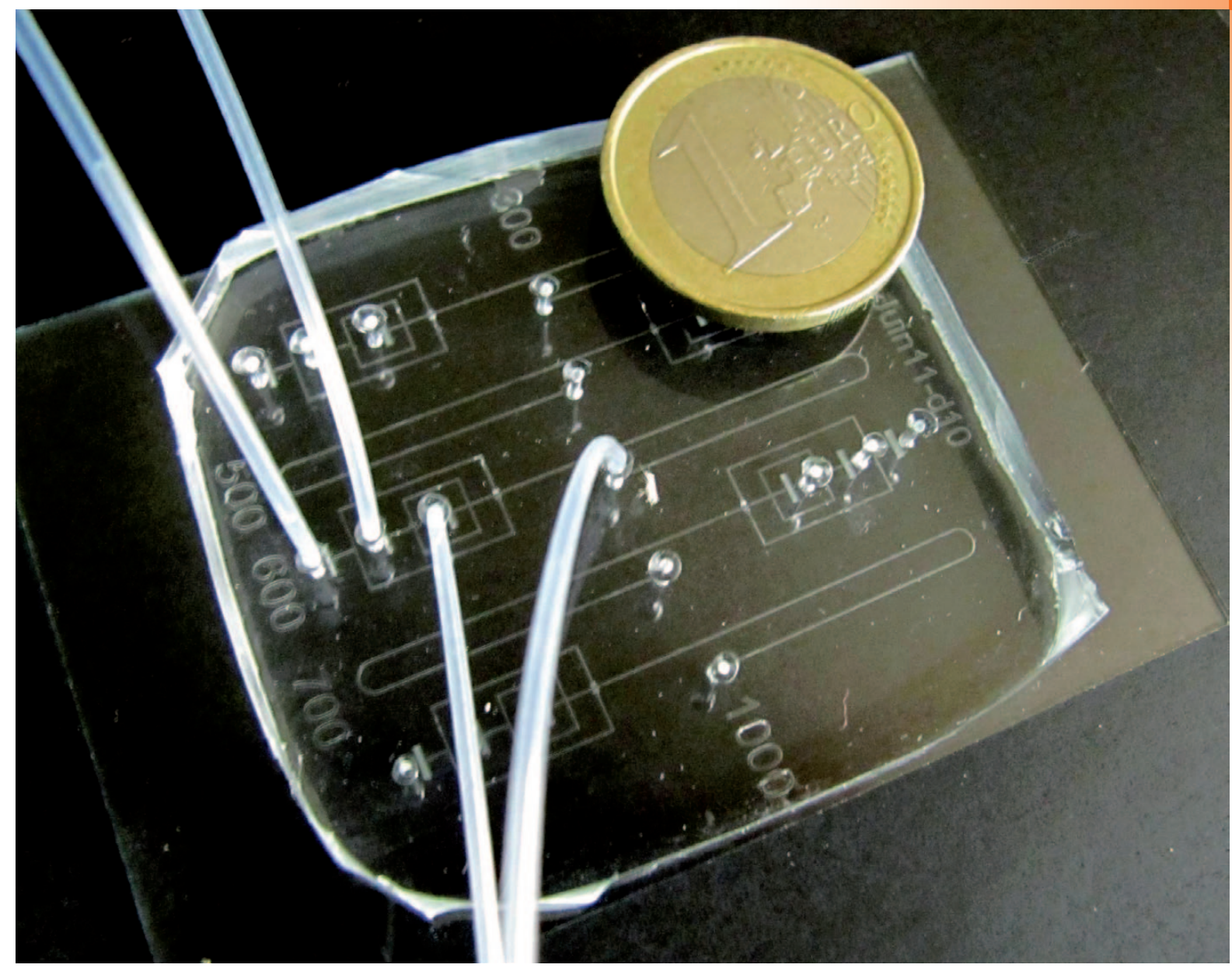

1. Puce microfluidique réalisée par lithographie douce. Les tubes connectés aux entrées et à la sortie du canal permettent la circulation des fluides. La pièce de un euro indique l'échelle de longueur.

\section{Analogie électricité linéaire - fluidique}

En régime laminaire stationnaire, le débit volumique $q$ d'un fluide newtonien s'écoulant dans un conduit indéformable de longueur $L$ varie linéairement avec $\Delta p$, la perte de charge (c'est-à-dire la différence de pression appliquée entre les deux extrémités de ce conduit), suivant une formule du type $q=\alpha \Delta p / \eta L$. Ici, $\eta$ est la viscosité du fluide et $\alpha$ est une grandeur s'exprimant en $\mathrm{m}^{4}$, qui dépend uniquement de la géométrie du conduit et des paramètres caractérisant sa section. Cette relation, connue dans la littérature sous le nom de formule de Poiseuille, est similaire à la relation d'Ohm qui traduit le transport de charges électriques dans un conducteur ohmique. De fait, il existe une analogie avec les circuits électriques linéaires, puisque des relations analogues aux lois de Kirchhoff des nœuds et des mailles s'appliquent aux réseaux microfluidiques : $q$ et $\Delta p$ sont les équivalents respectifs du courant et de la tension électriques. Pour cette raison, $R=\eta L / \alpha$, qui obéit aux mêmes règles d'association que les résistances électriques, est dénommée la résistance hydrodynamique du canal dans lequel s'écoule le fluide considéré. L'utilisation de cette analogie électricité - fluidique permet de déterminer très simplement les débits et les pertes de charges associés aux différents bras d'un réseau hydrodynamique (fig. E1).

\section{électricité linéaire}
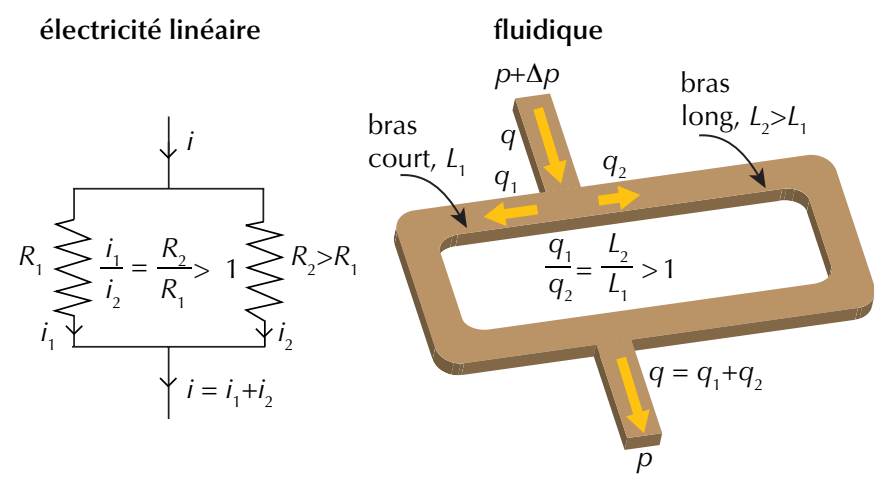

E1. Illustration de l'analogie électricité linéaire - fluidique. Une boucle asymétrique est un circuit fluidique, qui se comporte comme un diviseur de courant pour le débit d'un fluide newtonien. Les débits $q_{1}$ et $q_{2}$ des fluides s'écoulant dans les deux bras de la boucle vérifient la relation $q_{1} / q_{2}=R_{2} / R_{1}$, où $R_{1}$ et $R_{2}$ sont les résistances hydrodynamiques de ces deux bras de longueurs respectives $L_{1}$ et $L_{2}>L_{1}$. Pour des bras de même section, $q_{1} / q_{2}=L_{2} / L_{1}$. 
$>>$

réalisant une fonction de base : production, fusion, fragmentation, dilution, concentration, déformation de gouttes, etc (fig. 2).

Une puce de microfluidique digitale est donc une véritable chaîne d'assemblage micrométrique pour produire, par exemple, des trains alternés formés de gouttes de tailles ou de compositions différentes, des sphères ou des bâtonnets rigides produits par polymérisation photo-induite, des structures cœur-coquille (sphères rigides contenant un cœur liquide) ou des émulsions doubles (gouttes encapsulant plusieurs gouttelettes).

Une autre utilisation importante de la microfluidique digitale est basée sur l'équivalence entre l'espace et le temps existant pour des gouttes s'écoulant à vitesse constante $v$ : la visualisation de l'écoulement à une position $x=v t$ est équivalente à une mesure effectuée à un temps $t=x / v$. Cette spécificité ouvre la voie à la mise au point de procédés d'analyse rapide, autrefois limités à un résultat par tube à essai et aujourd'hui réalisés en flux continu. Ces caractéristiques sont bien évidemment largement utilisées pour le suivi de réactions chimiques; elles permettent la mesure de champs de concentration et l'estimation de cinétiques chimiques. Ces applications requièrent toutefois d'être capable de mélanger rapidement plusieurs fluides à l'intérieur de gouttes utilisées comme de véritables "microtubes à essai ». La recirculation interne dans les gouttes permet en fait d'obtenir un mélange rapide et efficace, notamment lorsque des canaux en forme de zigzag sont utilisés (fig. 2).

La maîtrise des opérations élémentaires de la microfluidique digitale nécessite de comprendre l'écoulement de gouttes au sein de réseaux hydrodynamiques. Il est possible de contrôler le trafic de façon active en pilotant des gouttes chargées à l'aide d'un champ électrique ou en les guidant avec une onde laser, mais nous n'évoquerons ici que des situations d'écoulements à débit imposé pour lesquels le trafic est uniquement régulé par les paramètres hydrodynamiques, le confinement des gouttes, l'espacement entre gouttes, et la géométrie du microcanal. Pour comprendre le trafic de gouttes dans des réseaux microfluidiques, il est d'abord essentiel de décrire leur circulation dans un canal simple.
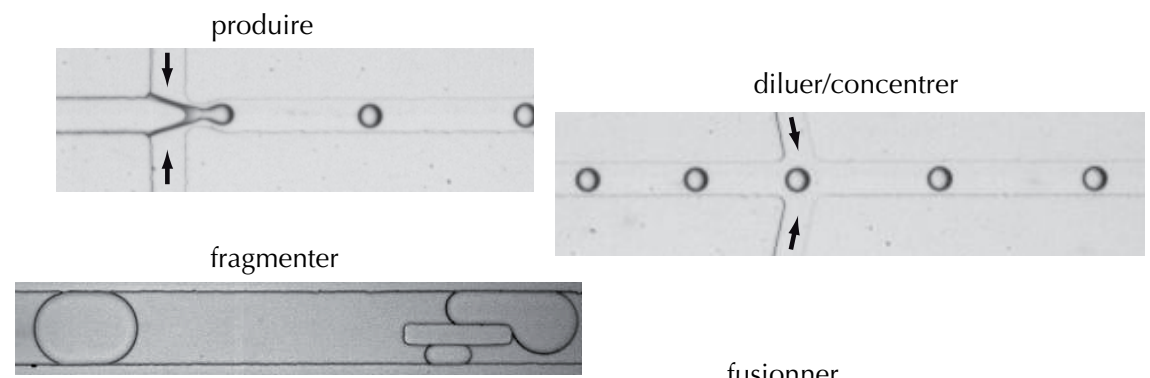

fusionner

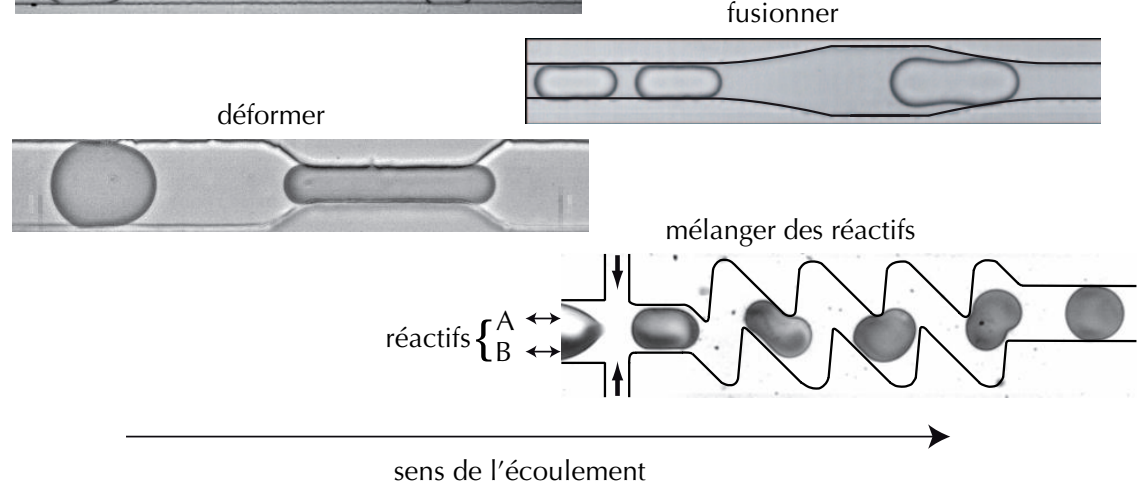

2. Opérations élémentaires en microfluidique digitale. La taille des objets dispersés (gouttes d'une phase aqueuse dans une huile) est de l'ordre de 10 à $100 \mu \mathrm{m}$. Les flèches indiquent les canaux d'injection de l'huile dans les images " produire », « diluer/concentrer » et " mélanger des réactifs ».

Nous remercions N. Brémond et F. Sarrazin pour la reproduction des images "fusionner » et " mélanger des réactifs ».

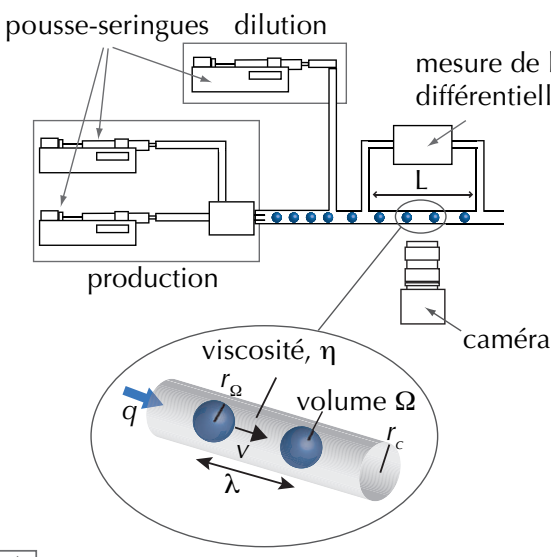

A

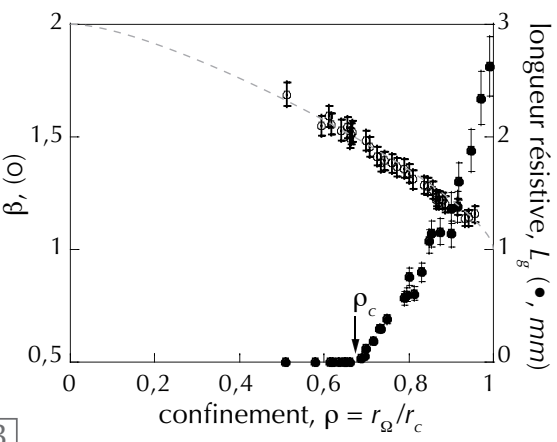

B

3. Étude expérimentale du transport de gouttes dans un canal de section constante.

(A) Montage utilisé pour étudier le transport de gouttes dans un conduit cylindrique de section $S_{c}=\pi r_{c}^{2}$ et de longueur L. Deux pousse-seringues injectent à débit constant la phase porteuse et la phase dispersée dans le module de production pour former un train périodique de gouttes monodisperses. Un supplément de phase porteuse dans le module de dilution contrôle la distance $\lambda$ entre gouttes et donc le nombre de gouttes $N=L / \lambda$. Le débit volumique total $q$ est la somme des débits des fluides dans les deux modules. Une caméra permet de déterminer $\lambda$ et la vitesse des gouttes $v$, et un capteur de pression mesure la pression différentielle $\Delta p$ sur la portion $L$ du canal. Les mesures de $v$ en fonction de $q / S$, et de $\Delta p / q$ en fonction de $\lambda^{-1}(=N / L)$ permettent de déterminer respectivement $\beta\left(=v S_{c} / q\right)$ et $L_{q}$ et d'étudier leurs variations en fonction de $\rho$.

(B) $\beta$ (cercles vides) et la longueur résistive $L_{q}$ (cercles pleins) sont fortement influencés par le degré de confinement des gouttelettes. La ligne en pointillés est un guide pour les yeux. On s’attend à ce que $1<\beta<2$. En effet, une goutte suffisamment petite pour ne pas perturber le profil parabolique de la vitesse du fluide porteur circule à une vitesse au plus égale à deux fois la vitesse moyenne de la phase porteuse, c'est-à-dire au plus égale à la vitesse maximale du fluide porteur. En revanche, lorsque $\rho=1$, le fluide porteur ne peut circuler autour de la goutte $: v$ est alors égale à la vitesse moyenne de la phase porteuse. (Figure adaptée de Belloul et al., Phys. Rev. Lett. 102 (2009) 194502.) 


\section{Transport de gouttes dans un conduit de section constante}

La perte de charge $\Delta p$ entre les deux extrémités d'un canal microfluidique et le débit volumique $q$ d'un fluide newtonien sont liés par la relation linéaire $\Delta p=R q$, où $R$ est la résistance hydrodynamique du conduit (voir encadré 1). La présence de gouttelettes dans ce conduit modifie l'écoulement de la phase continue et affecte donc $R$. Nous considérons des situations pour lesquelles la distance $\lambda$ entre gouttes est suffisamment grande pour que les régions où l'écoulement est modifié par les gouttes ne se superposent pas. En l'absence de couplages importants entre la forme de la goutte et l'écoulement (voir encadré 2), la résistance hydrodynamique d'un canal de longueur $L$ et de section $S_{c}$ varie linéairement avec le nombre de gouttes $N$ qu'il contient, et la vitesse de ces gouttes est proportionnelle au débit total $q$. Le transport des gouttes est alors décrit par deux relations :

$v=\beta q / S_{c}$ et $\Delta p / q=\left(L+N L_{g}\right) \eta / \alpha$, où $\beta$ et $L_{g}$ sont respectivement le rapport entre la vitesse $v$ des gouttes et la vitesse moyenne des deux fluides $q / S_{c}$, et une longueur résistive que rajoute chaque goutte à la portion du canal en terme de résistance hydrodynamique ; $\eta$ est la viscosité de la phase continue et $\alpha$ est une grandeur géométrique définie dans l'encadré 1 . Un conduit de longueur $L$ contenant $N$ gouttes, possède donc la même résistance hydrodynamique qu'un conduit de même section ne contenant pas de gouttes, mais ayant une longueur plus grande $L+N L_{g}$. Ces relations sont validées expérimentalement pour des canaux ayant des sections rectangulaires ou circulaires (fig. 3).

Les expériences dont le principe est décrit dans la figure $3 \mathrm{~A}$ et sa légende permettent de mesurer $\beta$ et $L_{g}$ et d'étudier leurs variations en fonction du confinement des gouttelettes. Les résultats obtenus mettent en lumière l'importance du confinement. Pour un tube cylindrique de rayon $r_{c}$ et des gouttes sphériques de rayon $r_{\Omega}<r_{c}$, il est par exemple possible de distinguer deux régimes suivant la valeur du paramètre $\rho=r_{\Omega} / r_{c}$ qui caractérise le confinement. En dessous d'une valeur critique $\rho_{c}$, les gouttes n'ajoutent aucune résistance hydrodynamique au canal dans lequel elles s'écoulent, donc $L_{g} \sim 0$, tandis que pour $\rho>\rho_{c}, L_{g}$ est une fonction croissante de $\rho$. Comme nous le verrons par la suite, ces résultats permettent de révéler les deux mécanismes physiques en compétition qui contrôlent le trafic de gouttes à une intersection.

\section{Sélection d'une route à une intersection}

\section{Une règle simple}

Une gouttelette arrivant à une intersection, par exemple une jonction en $\mathrm{T}$, est soumise à des forces de pression qui déterminent le canal de sortie dans lequel elle s'écoule. Prédire sa trajectoire est un problème difficile, qui nécessite la résolution numérique des champs tridimensionnels de vitesse et de pression à la jonction. Malgré la complexité de ce problème, une règle empirique simple permet toutefois de décrire remarquablement les phénomènes observés dans les dispositifs microfluidiques. Cette règle, dite $d u$ débit le plus fort, stipule que la goutte s'écoule toujours dans le canal où le débit instantané est le plus grand. Elle s'explique qualitativement par le fait que les gouttes produites sont situées au milieu du canal d'entrée de la jonction, et que les lignes de courant dans cette région partent majoritairement du côté du plus fort débit.

$\gg>$

\section{La microfluidique digitale, ou comment s'écoulent des gouttes confinées}

Une goutte peut changer de forme suivant la nature de l'écoulement auquel elle est soumise. Pour des écoulements laminaires, cette forme résulte d'une compétition entre les forces capillaires qui tendent à minimiser sa surface de contact avec le fluide environnant et les forces visqueuses, qui favorisent son élongation dans la direction de l'écoulement. Lorsque les effets visqueux sont négligeables devant les effets capillaires, comme cela est le cas pour les écoulements microfluidiques, la goutte adopte alors la forme d'équilibre qui minimise sa surface. Pour une goutte de volume $\Omega$ s'écoulant dans un conduit microfluidique, le confinement joue un rôle primordial pour la forme sélectionnée.

Dans le cas d'un conduit de section circulaire $\pi r_{c}^{2}$, le confinement peut être défini comme $\rho=r_{\Omega} / r_{C}$ avec $r_{\Omega}=(3 \Omega / 4 \pi)^{1 / 3}$ le rayon de la goutte, en supposant que sa forme est proche de celle $d^{\prime}$ une sphère (fig. E2A). Des gouttes très fortement confinées, $c^{\prime}$ est-à-dire pour lesquelles $\rho>1$, s'allongent dans le sens de l'écoulement.

Dans un conduit de section rectangulaire $h \times w$ pour lequel $h<w$, trois formes sont possibles suivant l'importance du confinement (fig. E2B). La goutte adopte une forme sphérique tant que son rayon $r_{\Omega}$ reste inférieur à $h / 2$. Lorsque la goutte est confinée verticalement, $r_{\Omega}>h / 2$, elle prend la forme d'un discoïde de hauteur $h$ et de rayon $r_{\Omega}^{\prime}$ de l'ordre de $(\Omega / h)^{1 / 2}$. Lorsque $r_{\Omega}^{\prime}>w / 2$, la goutte s'allonge dans le sens de l'écoulement. Dans le cas où $h<<w$, les gouttes sont fortement confinées verticalement $\left(\Omega \gg h^{3}\right)$ et on peut étudier l'influence du confinement latéral $2 r_{\Omega}^{\prime} / W$.

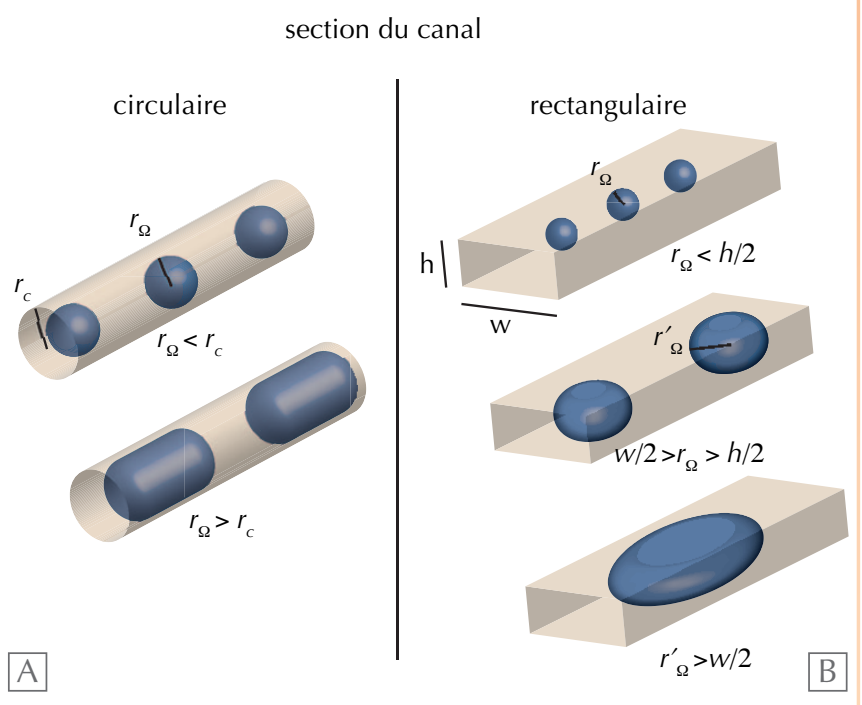

E2. Représentation schématique de l'effet du confinement sur la forme de gouttelettes s'écoulant dans des canaux ayant des sections (A) circulaires ou (B) rectangulaires. 
〉>

Voyage à travers

une boucle asymétrique

Considérons maintenant un train périodique de gouttelettes monodisperses, s'écoulant dans une boucle asymétrique constituée de deux bras de longueurs $L_{1}$ et $L_{2}>L_{1}$, dont les sections sont choisies identiques pour simplifier le problème, et intéressonsnous au trafic de ces gouttes (fig. 4A). Pour traiter cette question expérimentalement, on génère un train périodique de gouttes et on contrôle la distance $\lambda$ entre gouttes en associant un des modules de production précédemment décrits avec un module de dilution. Le train ainsi formé est dirigé vers l'entrée de la boucle. Lorsque les gouttes sont suffisamment éloignées, elles s'écoulent toutes dans le bras court, la boucle agissant alors dans ce régime comme un véritable filtre passif pour les gouttes. Si l'on concentre alors le train de gouttes, en dessous d'une valeur critique de $\lambda$, les gouttes se répartissent dans les deux bras (voir les transitions (1) et (2) définies dans la figure 4B). La transition entre régimes filtre et de répartition est relativement aisée à comprendre à l'aide des ingrédients élémentaires introduits auparavant.

\section{Une histoire de rétroactions hydrodynamiques}

Commençons par considérer la situation de gouttes fortement confinées $\left(\rho>\rho_{c}\right)$ et tentons de comprendre la transition (1) (fig. 4B). Pour des trains suffisamment dilués, lorsqu'une goutte se présente à l'entrée de la boucle, celles qui la précèdent en sont toutes sorties. Dans cette configuration, pour laquelle les deux bras ne contiennent aucune goutte, la boucle agit comme un diviseur de débit avec $q_{1}>q_{2}$ (voir encadré 1) et la règle du débit le plus fort indique que la goutte s'écoule toujours dans le bras court. Ce scénario se reproduit à l'identique pour chaque goutte arrivant à la jonction : cette situation correspond donc au régime filtre, pour lequel toutes les gouttes circulent au sein du bras court. Si l'on concentre peu à peu le train, lorsqu'une goutte se présente à l'entrée de la boucle, il est possible que toutes les gouttes la précédant ne soient pas encore sorties du bras court. Dans ce cas, leur présence dans ce bras de longueur $L_{1}$ entraîne une augmentation de sa résistance hydrodynamique, et donc une diminution en proportion du débit du fluide $q_{1}$. Si le nombre de ces gouttes est suffisant, la résistance hydrodynamique du bras court peut alors devenir supérieure à la résistance du bras long sans goutte, de sorte que $q_{1}<q_{2}$; la goutte suivante arrivant à la jonction s'écoulera alors dans le bras long de longueur $L_{2}$. Cette condition sur les résistances hydrodynamiques permet d'établir que la transition entre régimes filtre et de répartition se produit pour une valeur critique de $\lambda, \lambda_{\mathrm{f}}(\rho)$ (fig. $4 \mathrm{~B}$ ), qui varie comme $L_{g} /\left(L_{2} / L_{1}-1\right)$. En dessous de cette dilution critique, les gouttes se répartissent à la jonction en fonction des entrées et sorties des gouttes dans les deux bras de la boucle.

\section{Gare aux collisions entre gouttes !}

Le mécanisme précédent ne peut cependant pas expliquer la présence de gouttes dans le bras long observée pour $\rho<\rho_{c}$ lors de la transition (2) (fig. 4B). En effet, lorsque $\rho<\rho_{c}$, la figure 3B montre que $L_{g} \sim 0$ : les gouttes ne peuvent donc pas contribuer aux rétroactions hydrodynamiques, puisque leur présence n'affecte pas la résistance hydrodynamique du canal.
On s'attend donc à observer un régime filtre pour toute valeur de $\lambda$ dans cette gamme de confinements, ce qui de façon surprenante n'est pas le cas (fig. 4B). Les expériences révèlent que la transition filtre/répartition trouve ici son origine dans l'existence de collisions entre deux gouttes successives au nœud d'entrée de la boucle (fig. 4B). Suite à la collision entre deux gouttes, chacune d'entre elles se dirige en effet vers des bras différents : ces collisions remplacent ainsi les rétroactions hydrodynamiques en tant que mécanisme régulant le trafic pour les faibles dilutions. L'apparition de collisions pour les faibles dilutions s'explique simplement lorsque $\rho<\rho_{c}$. Dans le régime filtre, un temps $t$ est nécessaire pour qu'une goutte arrivée à la jonction se déplace d'une distance égale à son rayon vers le bras court. Lorsque $\lambda$ diminue, $t$ peut devenir comparable au temps $\tau$ séparant les arrivées de deux gouttes successives, ce qui conduit à l'apparition de collisions. L'existence de collisions est en fait un phénomène générique qui se 
produit quel que soit le degré de confinement des gouttes, pourvu que celles-ci soient suffisamment proches.

\section{Une dynamique complexe}

En l'absence de collisions, le trafic dans une boucle asymétrique est un système dynamique à retard, puisque le chemin sélectionné par une goutte dépend des trajets des gouttes précédentes. Les retards dans ce système sont liés aux temps de résidence d'une goutte dans les deux bras de la boucle et entraînent l'émergence de dynamiques complexes : les gouttes se répartissent dans la boucle de manière périodique, mais non triviale (fig. 5). Malgré la complexité de ce phénomène, il est possible d'établir un modèle analytique décrivant la dynamique discrète de la variable binaire caractérisant la sélection du chemin suivi à l'intersection (fig. 5A). Le trafic à travers la boucle est ainsi un système dynamique discret régi par l'itération de règles simples, un véritable automate cellulaire sur puce!
L'étude numérique et expérimentale du problème montre l'existence de deux quantités invariantes qui décrivent le système : les nombres $N_{p}$ de paquets et $N_{c}$ de gouttes par cycle (fig. 5A); un paquet correspond au nombre de gouttes successives circulant dans le bras court entre deux gouttes empruntant le chemin long. La période d'un cycle, c'est-à-dire le nombre de gouttes par cycle en unité de temps $\tau$, correspond à un des temps de résidence d'une goutte (dans le bras court ou dans le bras long) ou à la différence de ces temps de résidence. Le modèle permet de déterminer les règles de sélection de ces quantités invariantes et de décrire leurs évolutions en fonction des paramètres du problème : $\lambda, L_{g}, L_{1}$ et $L_{2}$. Enfin, les expériences mettent en lumière le rôle déterminant joué par le bruit (fluctuations de $\lambda$ ou de $L_{g}$ ), inhérent à toute expérience, provoquant des transitions entre plusieurs régimes périodiques, tous stables et qui se succèdent (fig. 5B).
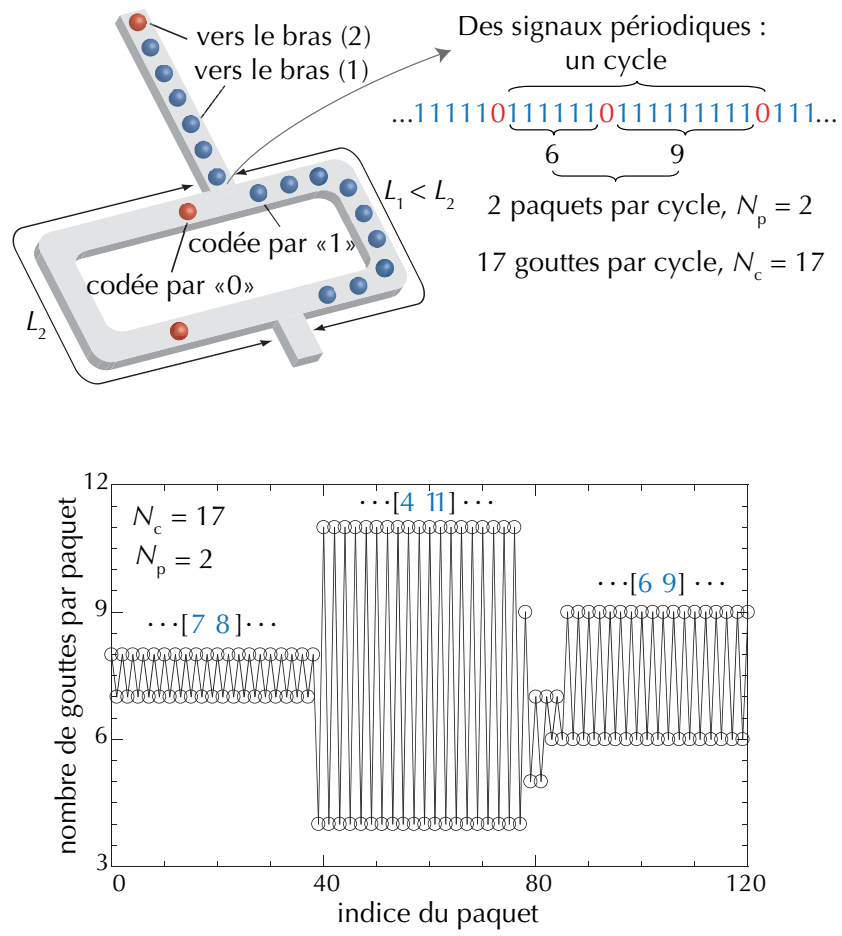

5. Une dynamique complexe de répartition des gouttes à une jonction.

(A) Schéma d'une boucle asymétrique utilisée pour étudier la dynamique de répartition des gouttes à une jonction. Le code couleur montre le chemin suivi par les gouttes à la jonction : le rouge indique les gouttes codées par " 0 » se dirigeant dans le bras long, et le bleu désigne les gouttes codées par " 1 » qui circulent dans le bras court. Des signaux périodiques sont observés expérimentalement et numériquement. Deux quantités sont invariantes : les nombres de paquets et de gouttes par cycle, respectivement $N_{p}$ et $N_{c}$.

(B) Évolution expérimentale du nombre de gouttes par paquet : la réponse est complexe ; mais $N_{p}$ et $N_{c}$ sont conservés durant cette longue série temporelle. II pourrait exister, a priori, autant de motifs périodiques que de façons de combiner $N_{p}$ entiers dont la somme est $N_{c}-N_{p}$, les permutations cycliques des $N_{p}$ entiers produisant le même motif de répartition. (Figure adaptée de Sessoms et al., Phys. Rev. Lett. 105 (2010) 154 501).

\section{Conclusion}

Il est en général difficile d'établir des modèles simples qui décrivent la circulation d'objets discrets à une intersection. Par exemple, le trafic pédestre ou routier est régi par des prises de décisions humaines individuelles.

Dans ce contexte, le trafic microfluidique de gouttes apparaît comme un système modèle régi par un processus de rétroaction hydrodynamique et par des collisions entre gouttes aux intersections, deux mécanismes en compétition contrôlés par le confinement et la dilution des gouttes. Ce système présente des dynamiques de répartition complexes, qui résultent de l'itération de règles simples et de l'existence de rétroactions liées aux entrées et sorties des gouttes dans les différents bras du réseau. La répartition des gouttes à une simple jonction en $\mathrm{T}$ dévoile ainsi l'existence d'effets de multistabilité (une coexistence de plusieurs attracteurs pour un même jeu de paramètres), qui est une caractéristique fréquente des systèmes à retard, comme c'est le cas pour des modèles de neurones.

Les recherches actuelles se tournent vers l'étude du trafic dans des réseaux plus complexes qu'une simple boucle et des canaux dans lesquels les gouttes circulent sur plusieurs voies. Après avoir sillonné les routes départementales, les gouttes empruntent désormais les autoroutes !

Ont participé aux travaux présentés dans cet article : Armand Ajdari, Malika Belloul, Annie Colin, Wilfried Engl, Matthieu Roché et David A. Sessoms, avec le soutien du CNRS, des Universités de Rennes 1 et de Bordeaux 1, de l'entreprise Rhodia, de la région Bretagne, de Rennes Métropole et du Fonds national suisse.

\section{En savoir plus}

- A. Ajdari, Reflets de la Physique n7 (2007), pp. 5-9.

- P. Tabeling, Introduction à la microfluidique, Belin (2003).

- M. Belloul et al., Phys. Rev. Lett., 102 (2009) 194502.

- D. A. Sessoms et al., Phys. Rev. Lett., 105 (2010) 154501. 